\title{
Solving Initial-Boundary Value Problems for Local Fractional Differential Equation by Local Fractional Fourier Series Method
}

\author{
Yu Zhang \\ College of Mathematics and Information Science, North China University of Water Resources and Electric Power, \\ Zhengzhou 450000, China
}

Correspondence should be addressed to Yu Zhang; b42old@163.com

Received 22 June 2014; Accepted 1 July 2014; Published 13 July 2014

Academic Editor: Xiao-Jun Yang

Copyright (C) 2014 Yu Zhang. This is an open access article distributed under the Creative Commons Attribution License, which permits unrestricted use, distribution, and reproduction in any medium, provided the original work is properly cited.

The initial-boundary value problems for the local fractional differential equation are investigated in this paper. The local fractional Fourier series solutions with the nondifferential terms are obtained. Two illustrative examples are given to show efficiency and accuracy of the presented method to process the local fractional differential equations.

\section{Introduction}

In various fields of physics, mathematics, and engineering, because of the different operators, there are classical differential equations [1], fractional differential equation [2-4], and local fractional differential equations $[5,6]$. There are more techniques to achieve analytical approximations to the solutions to differential equations in mathematical physics, such as the decomposition method [7], the variational iteration method [8], the homotopy perturbation method [9], the heatbalance integral method [10], the Fourier transform [11], the Laplace transform [11], and the references therein.

Recently, a new Fourier series (local fractional Fourier series) via local fractional operator was proposed [6] and had various applications in the applied fields such as fractal wave problems in fractal string $[12,13]$ and the heat-conduction problems arising in fractal heat transfer $[14,15]$. For a detailed description of the local fractional Fourier series method, we refer the readers to the recent works [14-16]. This is the main advantage of local fractional differential equations in comparison with classical integer-order and fractional-order models.

In the present paper we consider the local fractional differential equation:

$$
\frac{\partial^{\alpha} u(x, y)}{\partial y^{\alpha}}-\frac{\partial^{2 \alpha} u(x, y)}{\partial x^{2 \alpha}}=0
$$

subject to the initial-boundary value conditions:

$$
\frac{\partial^{\alpha} u(0, t)}{\partial x^{\alpha}}=0, \quad \frac{\partial^{\alpha} u(L, t)}{\partial x^{\alpha}}=0, \quad u(x, 0)=g(x),
$$

where the operators are described by the local fractional differential operators $[5,6,12-15]$. The paper is organized as follows. In Section 2, the basic theory of the local fractional calculus and local fractional Fourier series is presented. In Section 3, we discuss the initial-boundary problems for local fractional differential equation. Finally, Section 4 is devoted to the conclusions.

\section{Analysis of the Method}

In this section, we present the basic theory of the local fractional calculus and analyze the local fractional Fourier series method.

Definition 1. Let $F$ be a subset of the real line and be a fractal. The mass function $\gamma^{\alpha}[F, a, b]$ can be written as [5]

$$
\gamma^{\alpha}[F, a, b]=\lim _{0<i<n-1}\left(x_{i+1}-x_{i}\right) \rightarrow 0 \sum_{i=0}^{n-1} \frac{\left(x_{i+1}-x_{i}\right)^{\alpha}}{\Gamma(1+\alpha)} .
$$


The following properties are present as follows [5].

(i) If $F \cap(a, b)=\varnothing$, then $\gamma^{\alpha}[F, a, b]=0$.

(ii) If $a<b<c$ and $\gamma^{\alpha}[F, a, b]<0$, then $\gamma^{\alpha}[F, a, b]+$ $\gamma^{\alpha}[F, b, c]=\gamma^{\alpha}[F, a, c]$.

If $f:(F, d) \rightarrow\left(\Omega^{\prime}, d^{\prime}\right)$ is a bi-Lipschitz mapping, then we have $[5,12]$

$$
\rho^{s} H^{s}(F) \leq H^{s}(f(F)) \leq \tau^{s} H^{s}(F)
$$

such that

$$
\rho^{\alpha}\left|x_{1}-x_{2}\right|^{\alpha} \leq\left|f\left(x_{1}\right)-f\left(x_{2}\right)\right| \leq \tau^{\alpha}\left|x_{1}-x_{2}\right|^{\alpha} .
$$

In view of (5), we have

$$
\left|f\left(x_{1}\right)-f\left(x_{2}\right)\right| \leq \tau^{\alpha}\left|x_{1}-x_{2}\right|^{\alpha}
$$

such that

$$
\left|f\left(x_{1}\right)-f\left(x_{2}\right)\right|<\varepsilon^{\alpha},
$$

where $\alpha$ is the fractal dimension of $F$. This result is directly deduced from fractal geometry and relates to the fractal coarse-grained mass function $\gamma^{\alpha}[F, a, b]$, which reads $[5,13]$

$$
\gamma^{\alpha}[F, a, b]=\frac{H^{\alpha}(F \cap(a, b))}{\Gamma(1+\alpha)}
$$

with

$$
H^{\alpha}(F \cap(a, b))=(b-a)^{\alpha},
$$

where $H^{\alpha}$ is $\alpha$ dimensional Hausdorff measure.

Definition 2. If there is $[5,6,12-15]$

$$
\left|f(x)-f\left(x_{0}\right)\right|<\varepsilon^{\alpha}
$$

with $\left|x-x_{0}\right|<\delta$, for $\varepsilon, \delta>0$ and $\varepsilon, \delta \in R$, then $f(x)$ is called local fractional continuous at $x=x_{0}$.

If $f(x)$ is local fractional continuous on the interval $(a, b)$, then we can write it in the form $[5,6,12]$

$$
f(x) \in C_{\alpha}(a, b) .
$$

Definition 3. Local fractional derivative of $f(x)$ of order $\alpha$ at $x=x_{0}$ is defined as follows $[5,6,12-15]$ :

$$
f^{(\alpha)}\left(x_{0}\right)=\left.\frac{d^{\alpha} f(x)}{d x^{\alpha}}\right|_{x=x_{0}}=\lim _{x \rightarrow x_{0}} \frac{\Delta^{\alpha}\left(f(x)-f\left(x_{0}\right)\right)}{\left(x-x_{0}\right)^{\alpha}},
$$

where $\Delta^{\alpha}\left(f(x)-f\left(x_{0}\right)\right) \cong \Gamma(1+\alpha) \Delta\left(f(x)-f\left(x_{0}\right)\right)$.

From (12) we can rewrite the local fractional derivative as

$$
f^{(\alpha)}\left(x_{0}\right)=\lim _{x \rightarrow x_{0}} \frac{f(x)-f\left(x_{0}\right)}{\gamma^{\alpha}\left[F, x_{0}, x\right]},
$$

where

$$
\gamma^{\alpha}\left[F, x_{0}, x\right]=\frac{H^{\alpha}(F \cap(a, b))}{\Gamma(1+\alpha)} .
$$

Definition 4. The partition of the interval $[a, b]$ is $\left(t_{j}, t_{j+1}\right)$, $j=0, \ldots, N-1, t_{0}=a$ and $t_{N}=b$ with $\Delta t_{j}=t_{j+1}-t_{j}$ and $\Delta t=\max \left\{\Delta t_{1}, \Delta t_{2}, \Delta t_{j}, \ldots\right\}$. Local fractional integral of $f(x)$ of order $\alpha$ in the interval $[a, b]$ is given by $[5,6,12-15]$

$$
\begin{aligned}
{ }_{a} I_{b}^{(\alpha)} f(x) & =\frac{1}{\Gamma(1+\alpha)} \int_{a}^{b} f(t)(d t)^{\alpha} \\
& =\frac{1}{\Gamma(1+\alpha)} \lim _{\Delta t \rightarrow 0} \sum_{j=0}^{j=N-1} f\left(t_{j}\right)\left(\Delta t_{j}\right)^{\alpha} .
\end{aligned}
$$

Following (14), we have

$$
{ }_{a} I_{b}^{(\alpha)} f(x)=\frac{1}{\Gamma(1+\alpha)} \lim _{\Delta t \rightarrow 0} \sum_{j=0}^{j=N-1} f\left(t_{j}\right) \gamma^{\alpha}\left[F, t_{j}, t_{j+1}\right]
$$

where

$$
\gamma^{\alpha}[F, a, b]=\operatorname{limax}_{0<i<n-1}\left(x_{i+1}-x_{i}\right) \rightarrow 0 \sum_{i=0}^{n-1} \frac{\left(x_{i+1}-x_{i}\right)^{\alpha}}{\Gamma(1+\alpha)} .
$$

If $F$ are Cantor sets, we can get the derivative and integral on Cantor sets. follows:

Some properties of local fractional integrals are listed as

$$
\begin{gathered}
{ }_{0} I_{x}^{(\alpha)} E_{\alpha}\left(x^{\alpha}\right)=E_{\alpha}\left(x^{\alpha}\right)-1, \\
{ }_{0} I_{x}^{(\alpha)} \frac{x^{n \alpha}}{\Gamma(1+n \alpha)}=\frac{x^{(n+1) \alpha}}{\Gamma(1+(n+1) \alpha)}, \\
\sin _{\alpha}\left(a^{\alpha} x^{\alpha}\right)=\frac{1}{a^{\alpha}}\left[\cos _{\alpha}\left(a^{\alpha} x^{\alpha}\right)-1\right], \\
=-\frac{1}{a^{\alpha}}\left[\frac{x^{\alpha}}{\Gamma(1+\alpha)} \sin _{\alpha}\left(a^{\alpha} x^{\alpha}\right)\right. \\
{ }_{0} I_{x}^{(\alpha)} I_{x}^{(\alpha)} \cos _{\alpha}\left(a^{\alpha} x^{\alpha}\right)=\frac{1}{a^{\alpha}} \sin _{\alpha}\left(a^{\alpha} x^{\alpha}\right), \\
\Gamma(1+\alpha) \\
x^{\alpha} \cos _{\alpha}\left(a^{\alpha} x^{\alpha}\right) \\
=\frac{1}{a^{\alpha}}\left\{\frac{x^{\alpha}}{\Gamma(1+\alpha)} \sin _{\alpha}\left(a^{\alpha} x^{\alpha}\right)-\frac{1}{a^{\alpha}}\left[\sin _{\alpha}\left(a^{\alpha} x^{\alpha}\right)\right]\right. \\
{ }_{0} I_{x}^{(\alpha)}\left\{E_{x}^{(\alpha)}\left\{E_{\alpha}\left(x^{\alpha}\right) \sin _{\alpha}\left(a^{\alpha} x^{\alpha}\right)\right\}\right. \\
=\frac{\left.E_{\alpha}\left(x^{\alpha}\right)\left[\sin _{\alpha}\left(a^{\alpha} x^{\alpha}\right)-1\right]\right\}}{\left.\left.1+a^{\alpha}\right)-a^{\alpha} \cos _{\alpha}\left(a^{\alpha} x^{\alpha} x^{\alpha}\right)\right\}},
\end{gathered}
$$


Definition 5. Local fractional trigonometric Fourier series of $f(t)$ is given by $[6,12-16]$

$$
f(t)=a_{0}+\sum_{i=1}^{\infty} a_{k} \sin _{\alpha}\left(k^{\alpha} \omega_{0}^{\alpha} t^{\alpha}\right)+\sum_{i=1}^{\infty} b_{k} \cos _{\alpha}\left(k^{\alpha} \omega_{0}^{\alpha} t^{\alpha}\right)
$$

for $x \in R$ and $0<\alpha \leq 1$.

The local fractional Fourier coefficients of (19) can be computed by

$$
\begin{aligned}
& a_{0}=\frac{1}{T^{\alpha}} \Gamma(1+\alpha)_{0} I_{T} f(t), \\
& a_{k}=\left(\frac{2}{T}\right)^{\alpha} \Gamma(1+\alpha)_{0} I_{T}\left\{f(t) \sin _{\alpha}\left(k^{\alpha} \omega_{0}{ }^{\alpha} t^{\alpha}\right)\right\}, \\
& b_{k}=\left(\frac{2}{T}\right)^{\alpha} \Gamma(1+\alpha)_{0} I_{T}\left\{f(t) \cos _{\alpha}\left(k^{\alpha} \omega_{0}{ }^{\alpha} t^{\alpha}\right)\right\} .
\end{aligned}
$$

If $\omega_{0}=1$, then we get

$$
f(t)=a_{0}+\sum_{i=1}^{\infty} a_{k} \sin _{\alpha}\left(k^{\alpha} t^{\alpha}\right)+\sum_{i=1}^{\infty} b_{k} \cos _{\alpha}\left(k^{\alpha} t^{\alpha}\right),
$$

where the local fractional Fourier coefficients can be computed by

$$
\begin{aligned}
& a_{0}=\frac{1}{T^{\alpha}} \Gamma(1+\alpha)_{0} I_{T} f(t), \\
& a_{k}=\left(\frac{2}{T}\right)^{\alpha} \Gamma(1+\alpha)_{0} I_{T}\left\{f(t) \sin _{\alpha}\left(k^{\alpha} t^{\alpha}\right)\right\}, \\
& b_{k}=\left(\frac{2}{T}\right)^{\alpha} \Gamma(1+\alpha)_{0} I_{T}\left\{f(t) \cos _{\alpha}\left(k^{\alpha} t^{\alpha}\right)\right\} .
\end{aligned}
$$

\section{The Initial-Boundary Problems for the Local Fractional Differential Equation}

In this section, we consider (1) with the various initialboundary conditions.

Example 6. The initial-boundary condition (2) becomes

$$
\frac{\partial^{\alpha} u(0, t)}{\partial x^{\alpha}}=0, \quad \frac{\partial^{\alpha} u(L, t)}{\partial x^{\alpha}}=0, \quad u(x, 0)=E_{\alpha}\left(x^{\alpha}\right) .
$$

Let $u=X Y$ in (1). Separation of the variables yields

$$
X Y^{(\alpha)}=Y X^{(2 \alpha)} .
$$

Setting

$$
\frac{Y^{(\alpha)}}{Y}=\frac{X^{(2 \alpha)}}{X}=-\lambda^{2 \alpha},
$$

we obtain

$$
\begin{gathered}
X^{(2 \alpha)}+\lambda^{2 \alpha} X=0, \\
Y^{(\alpha)}+\lambda^{2 \alpha} Y=0 .
\end{gathered}
$$

Hence, we have their solutions, which read

$$
\begin{gathered}
X=a \cos _{\alpha}\left(\lambda^{\alpha} x^{\alpha}\right)+b \sin _{\alpha}\left(\lambda^{\alpha} x^{\alpha}\right), \\
Y=c E_{\alpha}\left(-\lambda^{2 \alpha} y^{\alpha}\right) .
\end{gathered}
$$

Therefore, a solution is written in the form

$$
\begin{aligned}
u(x, y) & =X Y \\
& =E_{\alpha}\left(-\lambda^{2 \alpha} y^{\alpha}\right)\left(A \cos _{\alpha}\left(\lambda^{\alpha} x^{\alpha}\right)+B \sin _{\alpha}\left(\lambda^{\alpha} x^{\alpha}\right)\right),
\end{aligned}
$$

where $A=a c, B=b c$.

For the given condition

$$
\frac{\partial^{\alpha} u(0, t)}{\partial x^{\alpha}}=0,
$$

there is $B=0$, so that

$$
u(x, y)=A E_{\alpha}\left(-\lambda^{2 \alpha} y^{\alpha}\right) \cos _{\alpha}\left(\lambda^{\alpha} x^{\alpha}\right) .
$$

For the given condition

$$
\frac{\partial^{\alpha} u(L, t)}{\partial x^{\alpha}}=0,
$$

we obtain

$$
\begin{gathered}
\sin _{\alpha}\left(\lambda^{\alpha} x^{\alpha}\right)=0, \\
\lambda^{\alpha}=\left(\frac{m \pi}{L}\right)^{\alpha}, \quad m \in Z^{+} \cup 0 .
\end{gathered}
$$

Thus, from (33) we deduce that

$$
\begin{array}{r}
u(x, y)=A E_{\alpha}\left(-\left(\frac{m \pi}{L}\right)^{2 \alpha} y^{\alpha}\right) \cos _{\alpha}\left(\left(\frac{m \pi x}{L}\right)^{\alpha}\right), \\
m \in Z^{+} \cup 0 .
\end{array}
$$

To satisfy the condition (23), (34) is written in the form

$$
u(x, y)
$$

$$
=A_{0}+\sum_{m=1}^{\infty} A_{m} E_{\alpha}\left(-\left(\frac{m \pi}{L}\right)^{2 \alpha} y^{\alpha}\right) \cos _{\alpha}\left(\left(\frac{m \pi x}{L}\right)^{\alpha}\right) .
$$


Then, we derive

$$
A_{0}=\frac{\Gamma(1+\alpha)}{L^{\alpha}}\left(E_{\alpha}\left(L^{\alpha}\right)-1\right)
$$

$$
\begin{aligned}
& A_{m} \\
& =\left(\frac{2}{L}\right)^{\alpha} \Gamma(1+\alpha) E_{\alpha}\left(x^{\alpha}\right) \\
& \quad \times \frac{\left[\cos _{\alpha}\left((m \pi / L)^{\alpha} x^{\alpha}\right)+(m \pi / L)^{\alpha} \sin \left((m \pi / L)^{\alpha} x^{\alpha}\right)\right]-1}{1+(m \pi / L)^{2 \alpha}}, \\
& u(x, y) \\
& =\frac{\Gamma(1+\alpha)}{2 L^{\alpha}}\left(E_{\alpha}\left(L^{\alpha}\right)-1\right) \\
& +\sum_{m=1}^{\infty} \frac{\left[\cos _{\alpha}\left((m \pi / L)^{\alpha} x^{\alpha}\right)+(m \pi / L)^{\alpha} \sin \left((m \pi / L)^{\alpha} x^{\alpha}\right)\right]-1}{1+(m \pi / L)^{2 \alpha}} \\
& \quad \times E_{\alpha}\left(-\left(\frac{m \pi}{L}\right)^{2 \alpha} y^{\alpha}\right) \cos _{\alpha}\left(\left(\frac{m \pi x}{L}\right)^{\alpha}\right) \\
& \quad \times E_{\alpha}\left(x^{\alpha}\right)\left(\frac{2}{L}\right)^{\alpha} \Gamma(1+\alpha) .
\end{aligned}
$$

Example 7. Let us consider (1) with the initial-boundary value condition, which becomes

$$
\frac{\partial^{\alpha} u(0, t)}{\partial x^{\alpha}}=0, \quad \frac{\partial^{\alpha} u(L, t)}{\partial x^{\alpha}}=0, \quad u(x, 0)=\frac{x^{\alpha}}{\Gamma(1+\alpha)}
$$

Following (35), we have

$$
\begin{aligned}
& u(x, y) \\
& \quad=A_{0}+\sum_{m=1}^{\infty} A_{m} E_{\alpha}\left(-\left(\frac{m \pi}{L}\right)^{2 \alpha} y^{\alpha}\right) \cos _{\alpha}\left(\left(\frac{m \pi x}{L}\right)^{\alpha}\right),
\end{aligned}
$$

where

$$
\begin{gathered}
A_{0}=\frac{1}{L^{\alpha}} \frac{\Gamma(1+\alpha)}{\Gamma(1+2 \alpha)} x^{2 \alpha}, \\
A_{m}=\frac{1}{(2 m \pi)^{\alpha}}\left\{\frac{x^{\alpha}}{\Gamma(1+\alpha)} \sin _{\alpha}\left(\left(\frac{m \pi x}{L}\right)^{\alpha}\right)\right. \\
\left.-\left(\frac{L}{m \pi}\right)^{\alpha}\left[\cos _{\alpha}\left(\left(\frac{m \pi x}{L}\right)^{\alpha}\right)-1\right]\right\} .
\end{gathered}
$$

Hence, we get

$$
\begin{aligned}
& u(x, y) \\
& =\frac{1}{L^{\alpha}} \frac{\Gamma(1+\alpha)}{\Gamma(1+2 \alpha)} x^{2 \alpha} \\
& \quad+\sum_{m=1}^{\infty} \frac{1}{(2 m \pi)^{\alpha}}\left\{\frac{x^{\alpha}}{\Gamma(1+\alpha)} \sin _{\alpha}\left(\left(\frac{m \pi x}{L}\right)^{\alpha}\right)\right. \\
& \left.\quad-\left(\frac{L}{m \pi}\right)^{\alpha}\left[\cos _{\alpha}\left(\left(\frac{m \pi x}{L}\right)^{\alpha}\right)-1\right]\right\} \\
& \quad \times E_{\alpha}\left(-\left(\frac{m \pi}{L}\right)^{2 \alpha} y^{\alpha}\right) \cos _{\alpha}\left(\left(\frac{m \pi x}{L}\right)^{\alpha}\right) .
\end{aligned}
$$

\section{Conclusions}

In this work, the initial-boundary value problems for the local fractional differential equation are discussed by using the local fractional Fourier series method. Analytical solutions for the local fractional differential equation with the nondifferentiable conditions are obtained.

\section{Conflict of Interests}

The author declares that there is no conflict of interests regarding the publication of this paper.

\section{References}

[1] A. M. Wazwaz, Partial Differential Equations: Methods and Applications, Balkema, Rotterdam, The Netherlands, 2002.

[2] A. A. Kilbas, H. M. Srivastava, and J. J. Trujillo, Theory and Applications of Fractional Differential Equations, Elsevier, Amsterdam, The Netherlands, 2006.

[3] I. Podlubny, Fractional Differential Equations, Academic Press, New York, NY, USA, 1999.

[4] J. Sabatier, O. P. Agrawal, and J. A. Tenreiro Machado, Advances in Fractional Calculus: Theoretical Developments and Applications in Physics and Engineering, Springer, New York, NY, USA, 2007.

[5] X. J. Yang, Advanced Local Fractional Calculus and Its Applications, World Science Publisher, New York, NY, USA, 2012.

[6] X. J. Yang, Local Fractional Functional Analysis and Its Applications, Asian Academic Publisher, Hong Kong, 2011.

[7] G. Adomian, Solving Frontier Problems of Physics: The Decomposition Method, Kluwer Academic, Dordrecht, The Netherlands, 1994.

[8] M. A. Noor and S. T. Mohyud-Din, "Variational iteration method for solving higher-order nonlinear boundary value problems using He's polynomials," International Journal of Nonlinear Sciences and Numerical Simulation, vol. 9, no. 2, pp. 141-156, 2008.

[9] H. Jafari and S. Momani, "Solving fractional diffusion and wave equations by modified homotopy perturbation method," Physics Letters. A, vol. 370, no. 5-6, pp. 388-396, 2007.

[10] J. Hristov, "Approximate solutions to fractional subdiffusion equations," European Physical Journal Special Topics, vol. 193, no. 1, pp. 229-243, 2011. 
[11] D. Baleanu, K. Diethelm, E. Scalas, and J. J. Trujillo, Fractional Calculus: Models and Numerical Methods, vol. 3 of Series on Complexity, Nonlinearity and Chaos, World Scientific, Boston, Mass, USA, 2012.

[12] M. Hu, R. P. Agarwal, and X. J. Yang, "Local fractional Fourier series with application to wave equation in fractal vibrating string," Abstract and Applied Analysis, vol. 2012, Article ID 567401, 15 pages, 2012.

[13] Y. Yang, D. Baleanu, and X. Yang, "Analysis of fractal wave equations by local fractional Fourier series method," Advances in Mathematical Physics, vol. 2013, Article ID 632309, 6 pages, 2013.

[14] Y. Z. Zhang, A. M. Yang, and X. J. Yang, "1-D heat conduction in a fractal medium: a solution by the local fractional Fourier series method," Thermal Science, vol. 17, no. 3, pp. 953-956, 2013.

[15] A. M. Yang, C. Cattani, C. Zhang et al., "Local fractional Fourier series solutions for non-homogeneous heat equations arising in fractal heat flow with local fractional derivative," 5, vol. 2014, Article ID 514639, 5 pages, 2014.

[16] Z. Y. Chen, C. Cattani, and W. P. Zhong, "Signal processing for nondifferentiable data defined on cantor sets: a local fractional fourier series approach," Advances in Mathematical Physics, vol. 2014, Article ID 561434, 7 pages, 2014. 


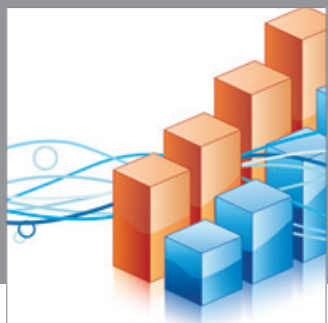

Advances in

Operations Research

mansans

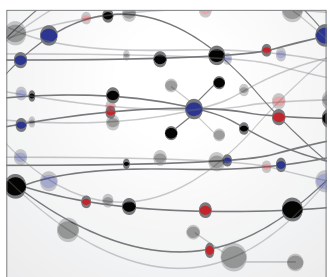

The Scientific World Journal
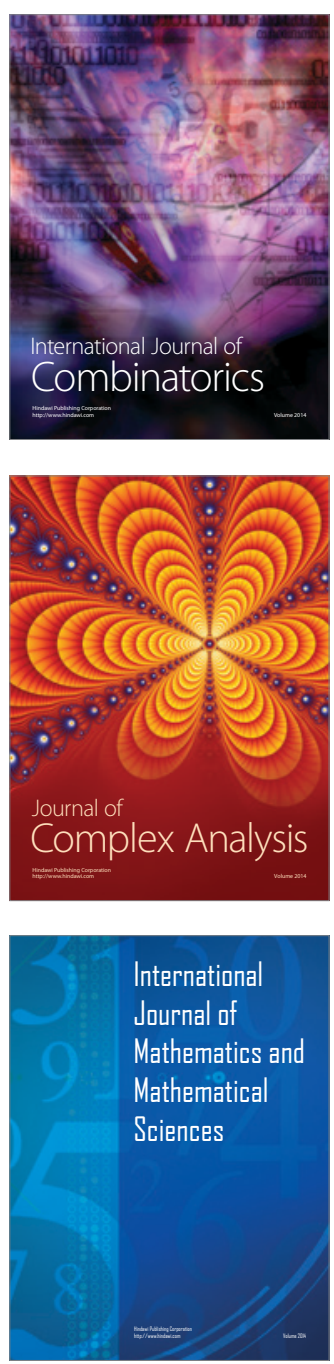
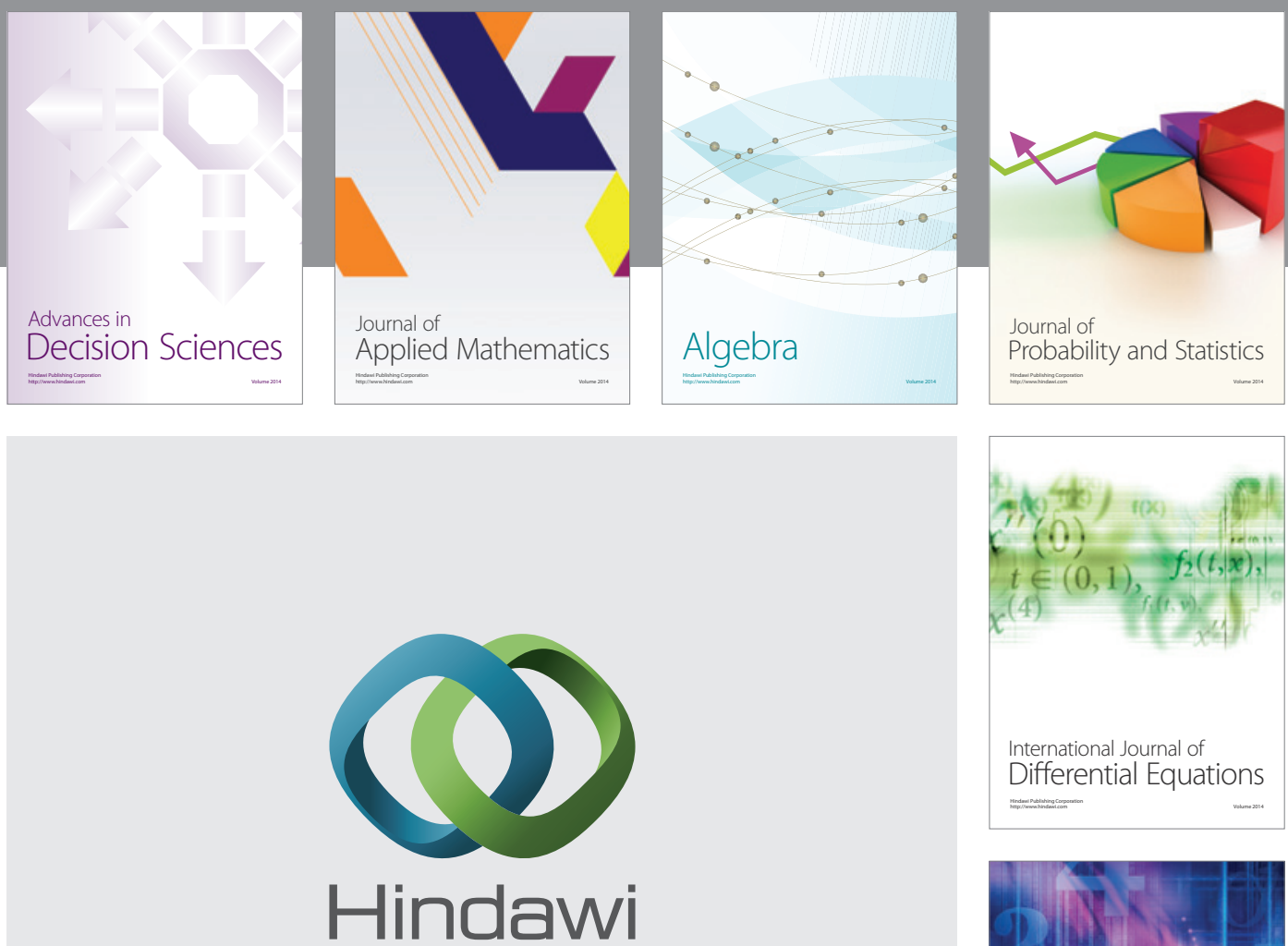

Submit your manuscripts at http://www.hindawi.com
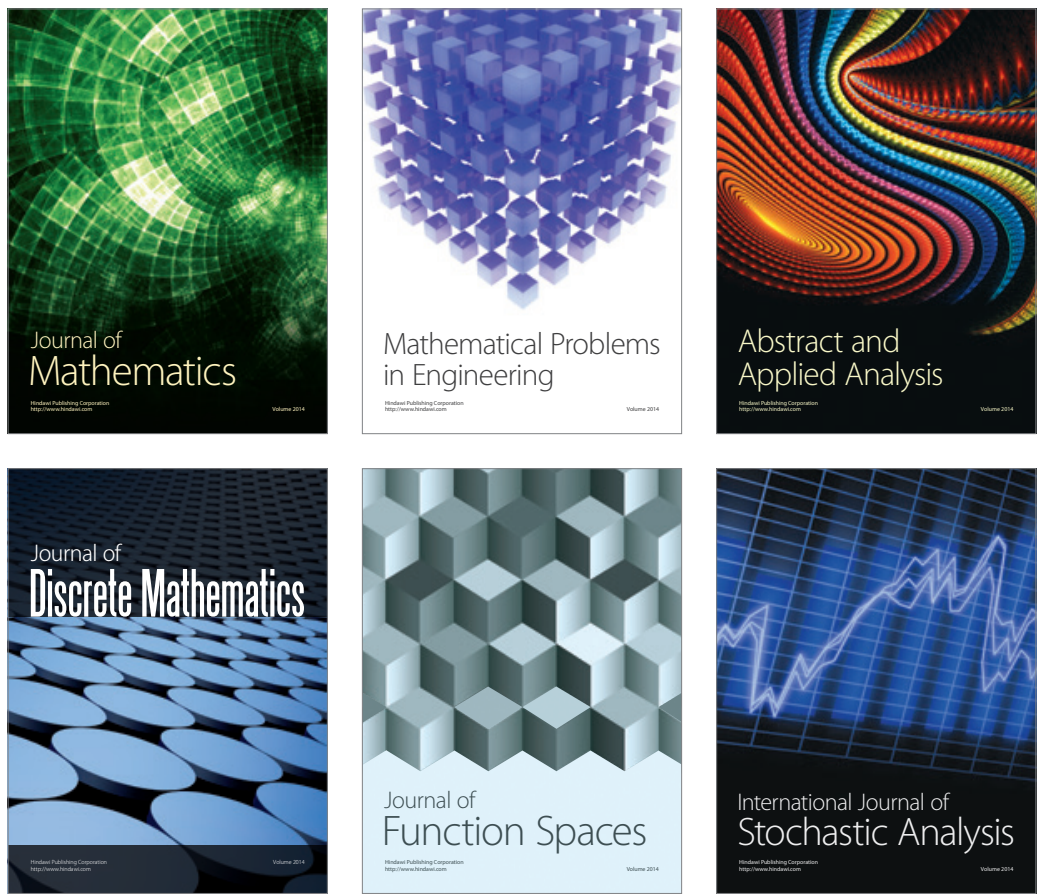

Journal of

Function Spaces

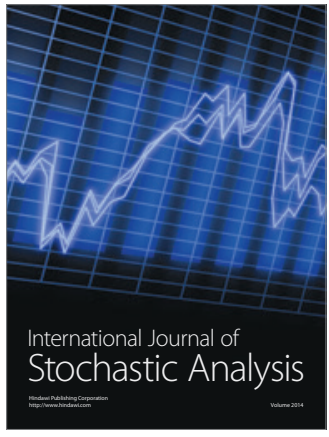

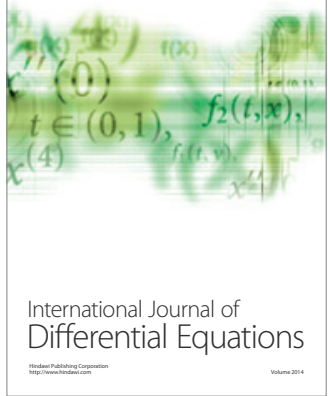
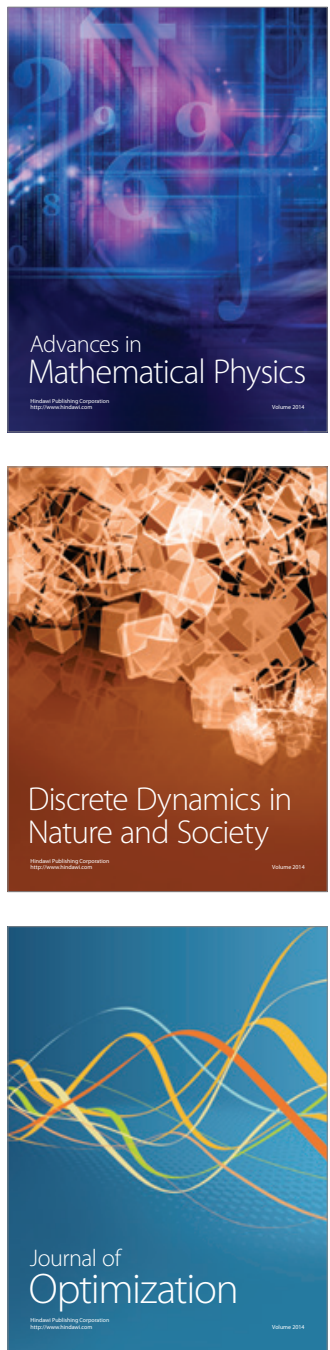